\title{
THE USE OF ANTI-MALARIAL DRUGS TO PREVENT MALARIA IN THE POPULATION OF MALARIA-ENDEMIC AREAS
}

\author{
BRIAN GREENWOOD \\ Department of Infectious and Tropical Disease, London School of Hygiene and Tropical Medicine, London, United Kingdom
}

\begin{abstract}
Anti-malarial drugs have been used in various ways to prevent malaria in the resident populations of endemic areas for nearly 100 years. The primary aim of most early studies was to interrupt transmission. This was rarely achieved, but administration of anti-malarial drugs either through medication of salt or by mass administration frequently led to a marked reduction in the prevalence of malaria infection and in the incidence of clinical attacks. Chemoprophylaxis is highly effective in reducing mortality and morbidity from malaria in young children and pregnant women living in endemic areas, but is difficult to sustain and, in some studies, has impaired the development of naturally acquired immunity. Intermittent preventive treatment, in which full therapeutic doses of a drug are given at defined intervals, has the potential to provide some of the benefits of sustained chemoprophylaxis in pregnant women and young children without some of its drawbacks and is a promising new approach to malaria control.
\end{abstract}

\section{INTRODUCTION}

The value of anti-malarial drugs in protecting short-term, non-immune visitors to malaria-endemic areas from the serious consequences of malaria infection is generally recognized but perhaps under-valued. Chemoprophylaxis has allowed a tourist industry to develop in some areas that were previously death traps for the non-immune, and prophylaxis has facilitated business travel and the economic development of many malaria-endemic areas. However, the use of anti-malarial drugs to protect the resident population of malaria-endemic areas has always proved to be much more controversial. In this paper, I review the various ways in which anti-malarial drugs have been used for this purpose in the past (Table 1) and discuss the current reawakening of interest in this approach to malaria control.

\section{ANTI-MALARIALS AND REDUCTION OF MALARIA TRANSMISSION}

The primary objective of most early studies of widespread distribution of anti-malarial drugs to the population of malaria endemic areas was interruption of malaria transmission. Two approaches have been tried: treatment of symptomatic cases and mass drug administration.

Treatment of symptomatic cases. The idea that treatment of symptomatic cases with an effective anti-malarial would reduce transmission and thus provide indirect protection to the population as a whole dates back to Robert Koch and the early years of the 20th century. ${ }^{1}$ Quinine was used extensively in Italy and elsewhere during the first part of the 20th century, partly with this aim in mind. However, quinine has little or no effect on gametocytes, a fact that was recognized by Celli, although he advocated extensive use of the drug. ${ }^{1}$ Thus, it is unlikely that treatment of cases with quinine had much impact on overall transmission of the infection. Treatment of clinical cases with a drug, such as an artemisinin, which kills gametocytes as well as asexual parasites, is more likely to be effective. Recent experience in Southeast Asia and in South Africa supports this view. On the Thai-Burmese border, replacement of mefloquine with mefloquine-artesunate as firstline treatment of patients with symptomatic malaria was associated with a substantial reduction in the incidence of Plasmodium falciparum infection, ${ }^{2}$ and widespread use of artemisinins may have contributed to a marked decline in the overall incidence of malaria in Vietnam. ${ }^{3}$ In South Africa, replacement of ineffective sulfadoxine-pyrimethamine (SP) with an artemisinin-based combination therapy (ACT) was again associated with a rapid decrease in the overall incidence of falciparum malaria. ${ }^{4}$ However, in Vietnam and in South Africa the introduction of artemisinins was associated with the introduction of other malaria control measures: insecticide treated nets (ITNs) in Vietnam and household residual spraying in South Africa. Thus, although these observations strongly suggest that introduction of ACTs for treatment of clinical cases had an effect on the overall level of malaria transmission in these low transmission areas, this has not been proven definitively.

Mass drug administration. In the highly endemic areas of Africa, in contrast to the situation in Southeast Asia and South Africa, most malaria infections are asymptomatic and go untreated, even when quite high levels of parasitemia are present. $^{5}$ In such situations, a significant reduction in the transmission of malaria by administration of a gametocidal drug is likely to be achieved only if asymptomatic as well as symptomatic subjects are treated. Detecting the latter requires a major investment in surveillance so the concept of mass drug administration (MDA) was developed. During an MDA, the whole population of a community known to contain a number of asymptomatic subjects is treated without determining who is infected.

Anti-malarials were distributed widely in endemic areas of Italy during the 1920 s and the 1930s, partly as personal prophylaxis but also to reduce transmission (Figure 1). Increased consumption of quinine was accompanied by a marked decrease in the incidence of malaria, ${ }^{1}$ but it is not certain that this was cause and effect. Quinine was also used extensively by Gorgas during the construction of the Panama Canal, in part as personal prophylaxis for canal workers, but also to reduce transmission (Figure 2 ). ${ }^{1}$ The use of MDAs to reduce transmission became a more promising approach to malaria control following the discovery of the 8-aminoquinolines, which are gametocidal One of the first studies to investigate the potential role of this class of drugs in interrupting transmission in an endemic area was a trial carried out in a rubber plantation in Liberia in 1930. Mass treatment of the population of two camps with plasmoquine led to a marked decrease in parasite prevalence and a reduction in the number of in- 
TABLE 1

Approaches to the administration of anti-malarial drugs as a means of preventing malaria in the population of malaria-endemic communities

Approach

Treatment of clinical cases

Medication of salt

Mass drug administration

Chemoprophylaxis

Intermittent preventive treatment
Comments

Requires that a high proportion of infections are symptomatic and that patients have easy access to treatment

Likely to be more effective if treatment includes a gametocidal drug

Produces sub-therapeutic but cumulative blood levels

Highly likely to induce resistance

Involves administration of drug, usually in therapeutic doses, to infected and non-infected subjects

Usually has only a transitory effect on levels of parasitemia and clinical malaria

Involves the repeated administration of drug, usually at sub-therapeutic doses, over a sustained period so as to obtain persistent protective blood levels

Involves the administration of a therapeutic dose of drug over an intermittent and defined period fected mosquitoes in the treated camps. ${ }^{6}$ During the $1960 \mathrm{~s}$ and 1970s, a number of trials of MDA were undertaken in Africa and Asia with the primary aim of interrupting transmission. These trials have recently been reviewed elsewhere. ${ }^{7}$ The results of most of these early trials are difficult to assess because they were not conducted in a way that would now be considered obligatory for a community-randomized trial; most involved before and after comparisons or comparisons of single intervention and unmatched control areas and some combined MDA with vector control. In nearly all cases, transmission was not interrupted although there was a marked reduction in parasite prevalence.

The most ambitious attempt to interrupt malaria transmission in a high transmission area of Africa using the MDA approach was undertaken in Garki in northern Nigeria in the 1970s. ${ }^{8}$ Household spraying with a residual insecticide was combined with several rounds of MDA with sulfalenepyrimethamine. Parasite and spleen rates decreased substantially in the intervention communities, but transmission was not interrupted and the intervention was generally considered to have failed. However, episodes of clinical malaria, and probably deaths, were reduced during the period of the intervention.

The largest MDA program reported was conducted in Nicaragua in 1981. A single round of treatment with chloroquine plus primaquine was given to approximately eight mil-

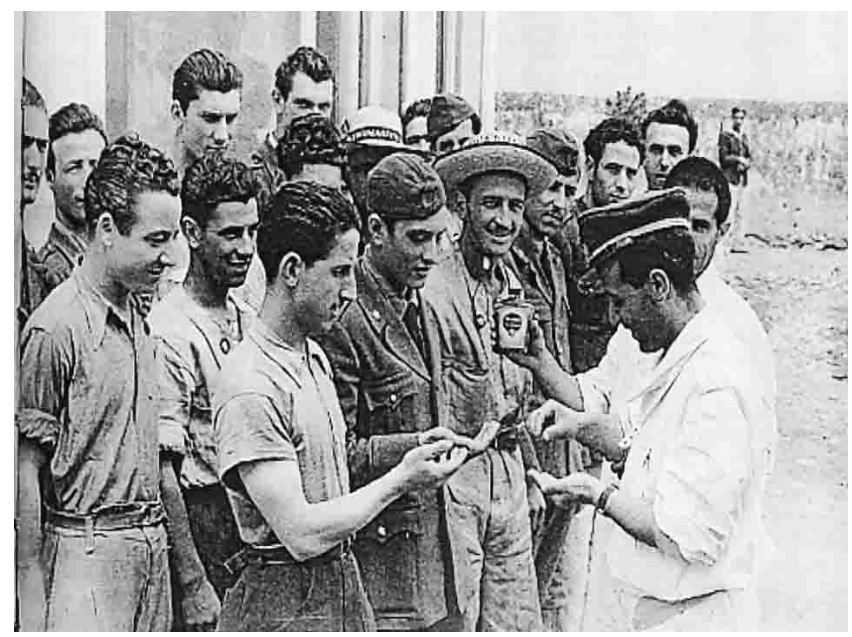

FIGURE 1. Distribution of antimalarials in Italy in the 1930s. (Archivio Casini, Sezione di storia della medicina, University of Rome 'La Sapienza'). lion people. ${ }^{9}$ There was a marked decrease in the incidence of malaria immediately after drug administration, but transmission was not interrupted and the incidence of malaria soon returned to its previous level.

A recent study of MDA was undertaken in The Gambia with the aim of delaying the onset of transmission in an area where malaria is highly seasonal and transmission limited to a few months of the year. ${ }^{10}$ Sulfadoxine-pyrimethamine and artesunate were given to the whole of the population of 33 villages at the end of the dry season when gametocyte levels are at their lowest. A reduction in the incidence of malaria compared with that seen in nine control villages where placebo was distributed was observed during the month after the administration of the drug combination, but no overall effect on the incidence of malaria throughout the course of the malaria transmission season was seen.

One success for MDA in achieving the goal of interruption of transmission has been reported recently from the island of Aneityum, Vanuatu. Eight rounds of MDA with chloroquineSP-primaquine combined with the introduction of ITNs and environmental control measures led to the elimination of falciparum infection from the island. ${ }^{11}$

In most MDAs, medication has been given in the form of tablets, sometimes under supervision. This ensures that an effective dose is given, but if large populations are to be covered on a repeated basis, this approach to drug delivery is demanding. In an attempt to overcome this problem, Pinotti devised the concept of drug delivery through the use of medicated salt. ${ }^{12}$ During the 1950 s and 1960s, a number of trials of medicated salt were undertaken in malaria-endemic areas. ${ }^{13}$ These generally achieved their objective of reducing the incidence of clinical episodes of malaria, but only at the cost of the rapid emergence of resistance. ${ }^{14,15}$ Studies in experimental systems have shown that exposure of malaria parasites to a sub-therapeutic dose of an anti-malarial over a prolonged period of time is the optimum way of inducing resistance. Use of medicated salt is likely to have a similar effect and has no place in malaria control.

Difficulties in delivery, problems with resistance, and the lack of a safe and highly effective anti-gametocidal drug limit the potential of MDAs in malaria control. MDAs may have a role in the final phase of a combined elimination strategy, as shown in Vanuatu. ${ }^{11}$ An MDA could also be useful in controlling an epidemic in a situation in which it is anticipated that transmission will decrease spontaneously within a short time, for example, after a period of flooding or a civil disturbance that leads to a short-term refugee situation. If an MDA 


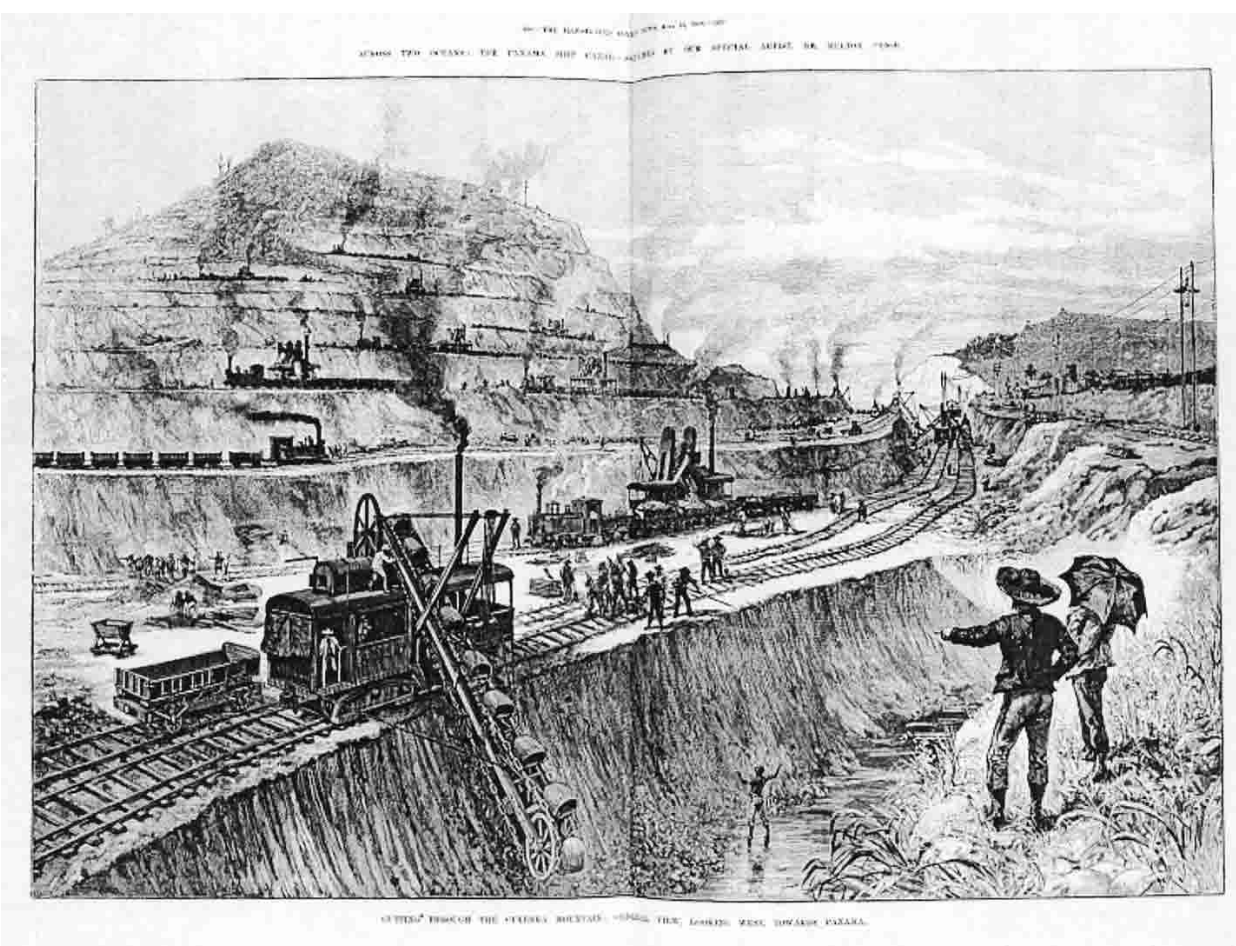

FIgure 2. Building the Panama Canal. (Wellcome Library, London).

is contemplated in such a situation, it should be given as soon as possible and the treatment regimen should incorporate a gametocidal drug, preferably an artemisinin combined with a longer acting partner.

\section{ANTI-MALARIALS AND THE PREVENTION OF MORTALITY AND MORBIDITY FROM MALARIA}

Introduction. Protection of the indigenous populations of malaria-endemic countries from malaria by regular administration of anti-malarial drugs has never found favor with the international malaria control community, although the operators of many mines and plantations have found it costeffective to protect their work force and their families from malaria in this way. Nevertheless, when chemoprophylaxis has been evaluated in this kind of situation, it has almost always been found to be highly effective in reducing morbidity and mortality from malaria, especially when targeted at high risk groups such as young children and pregnant women.

Chemoprophylaxis in children. In 1956, McGregor and others reported the results of a trial in The Gambia in which children were given chloroquine weekly from birth until the age of two years. ${ }^{16}$ Children who received chemoprophylaxis had fewer episodes of malaria, grew better, and had a higher mean hemoglobin and a lower gamma globulin concentration than children in the control group. These findings were reproduced in studies conducted subsequently in several other countries in Africa. ${ }^{17}$

A large trial of chemoprophylaxis in children less than five years of age was conducted in The Gambia in the early 1980s. ${ }^{18}$ More than 700 children were given chemoprophylaxis with Maloprim ${ }^{\circledR}$ (GlaxoSmithKline, Brentford, United kingdom) (pyrimethamine plus dapsone) by village health workers throughout the rainy season over a period of five years. Overall mortality in children who received prophylaxis was reduced by approximately 35\%. Protected children had fewer clinical attacks of malaria and a higher mean packed cell volume than control children. These results were sustained during several years of observation ${ }^{19}$ and are at least as impressive as those obtained with ITNs. A study conducted more recently in Tanzanian infants using the same antimalarial also showed a marked reduction in clinical attacks of malaria and in the incidence of severe anemia. ${ }^{20}$

Chemoprophylaxis in pregnant women. The first reported trial of chemoprophylaxis in pregnant women was undertaken in a mission hospital in western Nigeria in $1964 .^{21}$ This showed that chemoprophylaxis with pyrimethamine led to a substantial increase in birth weight in primigravidae and in grand-multiparous women. Subsequent studies, conducted in several countries in Africa, have confirmed the effect of chemoprophylaxis on birth weight ${ }^{22}$ and shown that it also results in an increase in maternal hemoglobin levels. ${ }^{23}$ Most studies have shown that the protective effect of chemoprophylaxis is most marked in first and second pregnancies. Drugs that have been used for chemoprophylaxis in pregnancy include chloroquine, pyrimethamine, proguanil, Maloprim $^{\circledR}$, and mefloquine. On the basis of the result of trials of chemoprophylaxis conducted in several countries in Africa, the recommendation of the World Health Organization was, until recently, that all pregnant women resident in areas of moderate or high malaria transmission should receive chemoprophylaxis with chloroquine throughout the second and third trimesters of pregnancy. However, this intervention has rarely been implemented on any significant scale.

Objections to the use of targeted chemoprophylaxis. Why, in the face of the impressive results discussed earlier, has 
chemoprophylaxis never been recommended for children resident in malaria-endemic countries or, in the case of pregnant women, recommended but rarely implemented? Some of the reasons why this is the case are now considered.

Sustainability. Sustaining compliance with prophylaxis is a difficult but not an insurmountable challenge. In The Gambia, one-third of villages sustained high levels of coverage with chemoprophylaxis in young children over a period of five years and another third achieved moderate levels of coverage. ${ }^{19}$ In this community, chemoprophylaxis was given by village health workers who received no financial support from the public health services and limited help from their communities. It is likely that higher levels of coverage could have been achieved if they had been paid. In most malaria-endemic countries, a high proportion of pregnant women attend an antenatal clinic at least once during pregnancy, but few attend often enough to allow the distribution of weekly chemoprophylaxis. Thus, if this approach to malaria control is to be followed, an alternative delivery system may be required. In The Gambia, this was provided effectively by traditional birth attendants. ${ }^{23}$

Safety. Drugs given on a regular basis to large numbers of healthy children or pregnant women must be extremely safe. No serious adverse events attributable to drug administration have been reported during trials of chemoprophylaxis in children, but the possibility that occasional serious adverse effects may have been missed cannot be excluded. In Sierra Leone, but not in The Gambia, chemoprophylaxis with Maloprim ${ }^{\circledR}$ resulted in hyperpigmented skin lesions, which disappeared after drug administration was stopped. ${ }^{24}$ There are few data on the safety of anti-malarials when used for chemoprophylaxis in pregnancy because most studies have involved only relatively small numbers of women and did not have the power to detect uncommon effects or a small increase in an adverse outcome of pregnancy. However, itching is one of the main reasons why chemoprophylaxis with chloroquine is unpopular among pregnant women.

Cost. Cost-effectiveness studies have shown that childhood chemoprophylaxis with a drug that costs approximately $\$ 0.1$ per administration and chemoprophylaxis in pregnancy are both highly cost effective. ${ }^{25}$ However, donor funds might be needed to introduce chemoprophylaxis on a national scale.

Impairment of the development of natural immunity. A major concern over the use of chemoprophylaxis in young children has been a fear that this will impair the development of natural immunity. Few studies have investigated this possibility directly. In The Gambia, children who received chemoprophylaxis for 1-5 years experienced an increase in clinical attacks of malaria during the year after chemoprophylaxis was stopped, but the effect was statistically significant only in those who had received anti-malarials from the ages of three months to five years. ${ }^{26}$ During the first year after chemoprophylaxis was stopped, there were 10 deaths in children who had received Maloprim ${ }^{\circledR}$ compared with four in a similar number of control children $(P=0.12)$, but survival curves showed that the protection from death obtained from chemoprophylaxis taken during early years of life was sustained up to 10 years of age. ${ }^{26}$ Because mortality rates decrease substantially after the age of five years, a very large trial involving tens of thousands of children would be needed to completely rule out a rebound mortality effect. A rebound effect was also observed during a more recent study in Tanzania. ${ }^{20}$ In this trial, the incidence of clinical malaria was significantly higher during the second and third years of life in children who had received chemoprophylaxis with Maloprim ${ }^{\circledR}$ during their first year of life than in a group of control children.

Primigravidae are believed to acquire some immunity to malaria through the acquisition of antibodies that interfere with the binding of $P$. falciparum to chondroitin sulfate in the placenta. Studies in Tanzania and in Kenya suggest that chemoprophylaxis can interfere with the development of these antibodies, ${ }^{27}$ and so if chemoprophylaxis is restricted to first or second pregnancies this could increase the risk of malaria in subsequent pregnancies. So far only one study has investigated this possibility. ${ }^{28}$ No reduction in birthweight or hemoglobin level was observed in secundigravidae who had received chemoprophylaxis during their first pregnancy compared with controls, but this study was small and a larger trial is needed to substantiate this finding.

Induction of drug resistance. It would be anticipated that widespread deployment of chemoprophylaxis in children and/ or pregnant women would increase the rate of spread of drug resistant parasites, the extent of the effect being related to the relative proportion of drug used for prophylaxis compared with the overall use of the drug in the community. The increase in drug use resulting from prophylaxis might be offset to some extent by a reduction in the number of clinical episodes of malaria requiring treatment. The use of medicated salt and the unrestricted use of chloroquine and pyrimethamine, including their use for prophylaxis, in the 1960s is likely to have contributed to the initial emergence and spread of resistance to these drugs. ${ }^{13}$ However, there is little information on whether the use of targeted chemoprophylaxis in children or pregnant women has had this effect. In a study from Tanzania, ${ }^{29}$ which is cited widely as evidence that this has occurred, prophylaxis with Maloprim ${ }^{\circledR}$ was followed within a few months by an increase in the prevalence of resistant parasites in children who were receiving prophylaxis, but also in control children resident in the same village who were not taking the drug. It seems unlikely that chemoprophylaxis in approximately $10 \%$ of the population for just a few months was responsible for replacement of most parasites within the village by resistant strains. It is more likely that this change was due to some other independent event. In The Gambia, no increase in pyrimethamine resistance was noted after five years of chemoprophylaxis with Maloprim ${ }^{\circledR}$ (Greenwood B, unpublished data). Chemoprophylaxis will inevitable lead to an increase in drug pressure, but this may be an acceptable price to pay if the benefits are substantial and it may be possible to reduce this risk by the use of combination therapy.

Role of chemoprophylaxis in current malaria control programs. The effectiveness of chemoprophylaxis in reducing mortality and morbidity from malaria in young children and in pregnant women has been demonstrated convincingly on many occasions, and the difficulties of implementing chemoprophylaxis and its potential risks have probably been exaggerated. Constraints on the deployment of chemoprophylaxis could be overcome in most cases using the increased financial resources that are now available for malaria control. However, increasing drug pressure, impairment of the development of natural immunity and difficulty in identifying a drug that is safe and effective remain problems for this approach to malaria control. Intermittent preventive treatment, which 
provides some of the benefits of chemoprophylaxis while reducing some of its risks, may be a more promising way forward.

\section{INTERMITTENT PREVENTIVE TREATMENT}

Introduction. Intermittent preventive treatment (IPT) describes the administration of a full therapeutic course of an anti-malarial to at risk subjects at specified times regardless of whether they are infected. IPT differs from chemoprophylaxis, which aims to sustain blood levels above the mean inhibitory concentration for a prolonged period, in producing protective drug concentrations for only short periods of time separated by periods when drug concentrations are below the level necessary to inhibit parasite growth. Because SP is the drug that has been used most widely for IPT in both pregnant women and children, it is not known whether IPT achieves its effect primarily by elimination of parasites or through the long-acting, prophylactic effect of SP.

IPT in pregnant women (IPT). Because of the difficulty in sustaining chemoprophylaxis in pregnant women, Schultz and others working in Malawi compared the effects of treatment with a full course of SP given twice during pregnancy with the effects of weekly chemoprophylaxis with chloroquine. ${ }^{30}$ The prevalence of low birth weight in the infants of women who received IPT with SP was significantly lower than that in women who received chemoprophylaxis with chloroquine. The protective effect against low birth weight of IPT with SP has subsequently been confirmed in larger effectiveness studies in Malawi, even when only one dose was given. ${ }^{31}$ In Kenya, a controlled trial showed that IPT with SP given two or three times during pregnancy reduced the prevalence of severe anemia in primigravidae and secundigravidae. ${ }^{32}$ However, a study in another area of Kenya showed that women infected with human immunodeficiency virus (HIV) required more than two or three doses of IPT to prevent infection of the placenta. ${ }^{33}$ On the basis of these rather limited results, the World Health Organization now recommends that IPT with SP, given at each antenatal clinic attendance after quickening, should replace chemoprophylaxis as the preferred chemotherapeutic method for the prevention of malaria in pregnancy. However, there are still a number of uncertainties over the use of IPTp. There are no data on the effectiveness of IPTp in areas of low endemicity or in areas where malaria transmission is highly seasonal. Because the mode of action of SP when used for IPTp is uncertain, it is not known whether in the increasing number of areas of Africa where SP is losing its efficacy, SP should be replaced with a long-acting drug such as mefloquine or whether a short acting drug such as Lapdap ${ }^{\circledR}$ (GlaxoSmithKline) (proguanil plus dapsone) would be as effective. The optimum approach to IPTp in women who are HIV-positive is not known. Thus, more research is needed if the full potential of this approach to the control of malaria in pregnancy is to be obtained.

IPT in infants (IPTi). The IPT concept has recently been applied to the prevention of malaria in infants. A study undertaken in Tanzania by Schellenberg and others ${ }^{34}$ showed that administration of a full dose of SP to infants at the time that they received their second and third doses of DPT and measles vaccines resulted in a $59 \%$ reduction in the incidence of clinical attacks of malaria and a $50 \%$ reduction in the incidence of severe anemia during the first year of life. A second study undertaken in Tanzania in which amodiaquine was given in a full therapeutic dose three times during the first year of life at the time of regular infant check-up clinics achieved similar impressive results. ${ }^{35}$ Importantly, there was no rebound in malaria attacks or in anemia during the second year of life in children who had received IPTi as infants. Trials of IPTi with SP are nearing completion in Ghana and Kenya, and further trials have started recently in Mozambique, Gabon, and Kenya. The results of these trials will help to define the epidemiologic conditions under which IPTi is effective. However, there are still many unanswered questions about the role of IPTi in malaria control. To address some of these important questions (Table 2), an IPTi consortium comprising several groups of investigators interested in this area of research, the World Health Organization, and the United Nations Children's Fund has recently been formed and has received support from the Bill and Melinda Gates Foundation.

IPT in children (IPTc). In high transmission areas, where a major proportion of deaths and severe morbidity from malaria occur during the first year of life, IPTi could make a major contribution to reducing the burden of malaria as a whole. However, in many areas of Africa, perhaps covering as much as $50 \%$ of the population at risk, the major burden of malaria is not in infants but in older children. This is especially the case in countries of the Sahel and sub-Sahel where malaria transmission is intense but very seasonal. In such areas, IPTi, even if highly effective, would have only a limited impact on the overall burden of malaria in children. Thus, a pilot study in Senegal is exploring whether the IPT principle can be applied to older children (Cisse B and others, unpublished data). In this study, SP and one dose of artesunate have been given to all eligible children less than five years old in a community with a total population of approximately 12,000 three times at one monthly intervals throughout the peak period of malaria transmission. This intervention resulted in a reduction in the incidence of clinical attacks of malaria of approximately $85 \%$. Observations are now under way to determine if this impressive effect is followed by a rebound. For the purpose of this trial, the anti-malarial drug was administered by project staff. Devising an effective method of delivery, which may be site specific, will be an important pre-

TABLE 2

Outstanding issues on the use of IPTi*

\footnotetext{
- Lack of knowledge of the efficacy of IPTi in different epidemiologic situations

- Lack of knowledge of the mode of action of SP when used for IPTi

- The possible impact of co-administration of an anti-malarial drug at the time of vaccination on the immune response to that vaccine

- Whether administration of an anti-malarial drug at the time of vaccination would enhance or detract from compliance with routine immunization

- The identity of drugs that could be used to replace SP for IPTi in areas of high SP resistance

- The safety of SP and alternative drugs when used on a large scale for IPTi

- The effect of IPTi on the development of natural immunity

- The cost efficacy of IPTi in different epidemiologic situations

- Whether IPTi could be readily implemented on a programmatic scale

* IPTi $=$ intermittent preventive treatment in infants; $\mathrm{SP}=$ sulfadoxine-pyrimethamine
} 
requisite for this approach to malaria control which is likely to be most effective in areas of intense, seasonal transmission where drug administration may be required on only two to four occasions each year.

\section{CONCLUSION}

The use of drugs to prevent malaria in the population of malaria-endemic areas has a long history. It has progressed from mass drug administration involving the whole population through sustained chemoprophylaxis targeted at risk groups to IPT in which drug administration is reduced to the minimal level required to achieve a useful protective effect. The use of anti-malarial drugs to prevent malaria will always involve balancing the level of protection achieved with the cost of the drug, its side effects, and its impact on the development of natural immunity and drug resistance. If the initial results of studies in infants and children are confirmed, the IPT approach may offer the optimum balance that can be achieved.

Acknowledgments: I thank David Schellenberg, Geoff Targett and Lorenz von Seidlein for their helpful comments on the manuscript. This paper is based on the Leon Jacobs Gorgas Memorial Lecture given at the National Institutes of Health, Bethesda, MD, on April 2, 2003.

Author's address: Brian Greenwood, Department of Infectious and Tropical Diseases, London School of Hygiene and Tropical Medicine, Keppel Street, London WC1E 7HT, United Kingdom, E-mail: brian.greenwood@lshtm.ac.uk.

\section{REFERENCES}

1. Harrison G, 1978. Mosquitoes, Malaria and Man; A History of the Hostilities Since 1880. London: John Murray.

2. Nosten F, van Vugt M, Price R, Luxemburger C, Thway KL, Brockman A, McGready R, ter Kuile F, Looareesuwan S, White NJ, 2000. Effects of artesunate mefloquine combination on incidence of Plasmodium falciparum and mefloquine resistance in western Thailand: a prospective study. Lancet 356: 297-302.

3. Hung LQ, de Vries PJ, Giao PT, Nam NV, Binh TQ, Chong MT, Quoc NTTA, Thanh TN, Hung LN, Kager PA, 2002. Control of malaria; a successful experience from Vietnam. Bull World Health Organ 80: 660-666.

4. Barnes KI, Durrheim DN, Jackson A, Mehta U, Tsoka J, Diamini SS, Qwabe B, Bredenkamp B, Nettleship E, Mthembu D, Mthembu DJ, Sharp BL, 2003. Epidemiology of malaria following implementation of artemether-lumefantrine as first-line treatment of uncomplicated disease in KwaZulu-Natal, South Africa. Antibiot Chemother 7: 6.

5. Von Seidlein L, Clarke S, Alexander N, Manneh F, Doherty T, Pinder M, Walraven G, Greenwood BM, 2002. Treatment uptake by individuals infected with Plasmodium falciparum in rural Gambia, West Africa. Bull World Health Organ 80: 790796.

6. Barber MA, Rice JB, Brown JY, 1932. Malaria studies on the Firestone rubber plantation in Liberia, West Africa. Am J Hyg 15: 601-623.

7. Von Seidlein L, Greenwood BM, 2003. Mass administration of antimalarial drugs. Trends Parasito 19: 452-460.

8. Molineaux L, Gramiccia G, 1980. The Garki Project. Geneva: World Health Organization.

9. Garfield RM, Vermund SH, 1983. Changes in malaria incidence after mass drug administration in Nicaragua. Lancet ii: 500 503.

10. Von Seidlein L, Walraven G, Milligan PLM, Alexander N, Manneh F, Deen JL, Coleman R, Jawara M, Lindsay SW, Drakeley C, De Martin S, Olliaro P, Bennett S, Schimm M, Okunoye K,
Targett GAT, McAdam KPWJ, Doherty JF, Greenwood BM, Pinder M, 2003. The effect of mass administration of pyrimethamine/sulphadoxine combined with artesunate on malaria transmission: a double blind, community randomised, placebo controlled trial in The Gambia. Trans $R$ Soc Trop Med Hyg 97: 217-225.

11. Kaneko A, Taleo G, Kalkoa M, Yamar S, Kobayakawa T, Björkman A, 2000. Malaria eradication on islands. Lancet 356: 1560 1564.

12. Pinotti M, 1954. Chemoprophylaxis of malaria by the association of an antimalarial drug to the sodium chloride used daily in the preparation of meals. Fifth International Congress of Tropical Medicine and Malaria, 1953. Volume 2. Instanbul, Turkey, $248-258$.

13. Payne D, 1988. Did medicated salt hasten the spread of chloroquine resistance in Plasmodium falciparum? Parasitol Today 4: $112-115$.

14. Meuwissen JHET, 1964. The use of medicated salt in an antimalaria campaign in West New Guinea. Trop Geogr Med 16: 245-255.

15. Giglioli G, Rutten FJ, Ramjattan S, 1967. Interruption of malaria transmission by chloroquinized salt in Guyana with observations on a chloroquine-resistant strain of Plasmodium falciparum. Bull World Health Organ 36: 283-301.

16. McGregor IA, Gilles HM, Walter JH, Davies AH, Pearson FA, 1956. Effects of heavy and repeated malaria infections on Gambian infants and children. Effects of erythrocytic parasitization. BMJ ii: 686-692.

17. Prinsen Geerlins PD, Brabin BJ, Eggelte TA, 2003. Analysis of the effects of malaria chemoprophylaxis in children on haematological responses, morbidity and mortality. Bull World Health Organ 81: 205-216.

18. Greenwood BM, Greenwood AM, Bradley AK, Snow RW, Byass P, Hayes RJ, N'Jie ABH, 1988. Comparison of two strategies for control of malaria within a primary health care programme in The Gambia. Lancet $i$ : 1121-1127.

19. Allen SJ, Snow RW, Menon A, Greenwood BM, 1990. Compliance with malaria chemoprophylaxis over a five year period among children in a rural are of the Gambia. J Trop Med Hyg 93: 313-322.

20. Menendez C, Kahigwa E, Hirt R, Vounatsou P, Aponte JJ, Font F, Acosta CJ, Schellenberg DM, Galindo CM, Kimario J, Urassa, Brabin B, Smith TA, Kitua AY, Tanner M, Alonso PL, 1997. Randomised placebo-controlled trial of iron supplementation and malaria chemoprophylaxis for prevention of severe anaemia and malaria in Tanzanian infants. Lancet 350: 844-850.

21. Morley D, Woodland M, Cuthbertson WFJ, 1964. Controlled trial of pyrimethamine in pregnant women in an African village. $B M J$ : $:$ : $67-68$.

22. Garner P, Gülmezoglu AM, 2001 Prevention Versus Treatment for Malaria in Pregnant Women (Cochrane Review). The Cochrane Library, Issue 4. Oxford: Update Software.

23. Greenwood BM, Greenwood AM, Snow RW, Byass P, Bennett S, Hatib N'Jie AB, 1989. The effects of malaria chemoprophylaxis given by traditional birth attendants on the course and outcome of pregnancy. Trans $R$ Soc Trop Med Hyg 83: 589-594.

24. David KP, Marbiah NT, Lovtren P, Greenwood BM, 1997. Hyperpigmented dermal macules in children following the administration of Maloprim ${ }^{\mathrm{R}}$ for malaria chemoprophylaxis. Trans $R$ Soc Trop Med Hyg 91: 204-208.

25. Goodman CA, Coleman PG, Mills AJ, 1999. Cost-effectiveness of malaria control in sub-Saharan Africa. Lancet 354: 378-385.

26. Greenwood BM, David PH, Otoo-Forbes L, Allen SJ, Alonso PL, Armstrong-Schellenberg JR, Byass P, Hurwitz M, Menon A, Snow RW, 1995. Mortality and morbidity from malaria after stopping malaria chemoprophylaxis. Trans $R$ Soc Trop Med Hyg 89: 629-633.

27. Staalsoe T, Megnekou R, Fievét N, Ricke CH, Zornig HD, Leke R, Taylor DW, Deloron P, Hviid L, 2001. Acquisition and decay of antibodies to pregnancy-associated variant antigens on the surface of Plasmodium falciparum-infected erythrocytes that protect against placental parasitemia. J Infect Dis 184: 618-626. 
28. Greenwood AM, Menendez C, Alonso PL, Jaffar S, Langerock P, Lulat S, Todd J, M'Boge B, Francis N, Greenwood BM, 1994. Can malaria chemoprophylaxis be restricted to first pregnancies? Trans $R$ Soc Trop Med Hyg 88: 681-682.

29. Lemnge MM, Msangeni HA, Rønn AM, Salum FM, Jakobsen PH, Mhina JI, Akida JA, Bygbjerg, 1997. Maloprim ${ }^{\mathrm{R}}$ malaria prophylaxis in children living in a holoendemic village in north-eastern Tanzania. Trans R Soc Trop Med Hyg 91: 68-73.

30. Schultz LJ, Steketee RW, Macheso A, Kazembe P, Chitsulo L, Wirima JJ, 1994. The efficacy of antimalarial regimens containing sulfadoxine-pyrimethamine and/or chloroquine in preventing peripheral and placental Plasmodium falciparum infection among pregnant women in Malawi. Am J Trop Med Hyg 51: 515-522.

31. Rogerson SJ, Chaluluka E, Kanjala M, Mkundika P, Mhango C, Molyneux M, 2000. Intermittent sulfadoxine-pyrimethamine in pregnancy: effectiveness against malaria morbidity in Blantyre, Malawi, in 1997-99. Trans $R$ Soc Trop Med Hyg 94: 549-553.

32. Shulman CE, Dorman EK, Cutts F, Kawuondo K, Bulmer JN,
Peshu N, Marsh K, 1999. Intermittent sulphadoxine-pyrimethamine to prevent severe anaemia secondary to malaria in pregnancy. Lancet 353: 632-636.

33. Parise ME, Ayisi JG, Nahlen BL, Schultz LJ, Roberts JM, Misore A, Muga R, Oloo AJ, Steketee RW, 1998. Efficacy of sulfadoxine-pyrimethamine for prevention of placental malaria in an area of Kenya with a high prevalence of malaria and human immunodeficiency virus infection. Am J Trop Med Hyg 59: 813-822.

34. Schellenberg D, Menendez C, Kahigwa E, Aponte JJ, Vidal J, Tanner M, Mshinda H, Alonso P, 2001. Intermittent treatment for malaria and anaemia control at time of routine vaccination in Tanzanian infants; a randomised, placebo-controlled trial. Lancet 357: 1471-1477.

35. Massaga JJ, Kitua AY, Lemnge M, Akida JA, Malle LN, Rønn AM, Theander TG, Bygbjerg IC, 2003. Effect of intermittent treatment with amodiaquine on anaemia and malaria fevers in Tanzania; a randomised, placebo-controlled trial. Lancet 361: 1853-1860. 